\title{
MOD 2 REPRESENTATIONS OF ELLIPTIC CURVES
}

\author{
K. RUBIN AND A. SILVERBERG
}

\begin{abstract}
Explicit equations are given for the elliptic curves (in characteristic $\neq 2,3$ ) with mod 2 representation isomorphic to that of a given one.
\end{abstract}

\section{INTRODUCTION}

If $N$ is a positive integer and $E$ is an elliptic curve defined over a field $F$, one can ask for a description of the set of elliptic curves whose mod $N$ representation (of the absolute Galois group) is symplectically isomorphic to that of $E$ (see [2]). For $N=3,4$, and 5, we gave explicit equations in [3] and [5]. The case $N=1$ is trivial, and when $N \geq 7$ the set in question is always finite and the situation is quite different from the ones we consider. In [4] we gave a description for $N=6$ (but did not give explicit equations).

This note, which can be viewed as a footnote to those papers, deals with the easier case $N=2$. Note that since there is only one nondegenerate alternating pairing on $\mathbf{Z} / 2 \mathbf{Z} \times \mathbf{Z} / 2 \mathbf{Z}$, isomorphic and symplectically isomorphic are the same for mod 2 representations. Theorem 1 gives explicit equations for the family of elliptic curves whose mod 2 representation is isomorphic to that of a given one. Given two elliptic curves, Corollary 2 gives an easy way to determine whether or not their mod 2 representations are isomorphic. The proofs are given in $\S 2$. In $\S 3$ we give a different approach, using the algorithm from [3].

If $F$ is a field, let $F^{\text {sep }}$ denote a separable closure of $F$ and let $G_{F}=\operatorname{Gal}\left(F^{\text {sep }} / F\right)$. If $E$ is an elliptic curve over $F$, let $j(E)$ denote its $j$-invariant, let $\Delta(E)$ denote its discriminant, and let $E[2]$ denote the $G_{F}$-module of 2-torsion points on $E$.

Theorem 1. Suppose $F$ is a field of characteristic different from 2 and 3 , and $E: y^{2}=x^{3}+a x+b$ is an elliptic curve over $F$. If $u, v \in F$, let $\mathcal{E}_{u, v}$ denote the curve

$y^{2}=x^{3}+3\left(3 a v^{2}+9 b u v-a^{2} u^{2}\right) x+27 b v^{3}-18 a^{2} u v^{2}-27 a b u^{2} v-\left(2 a^{3}+27 b^{2}\right) u^{3}$.

If $E^{\prime}$ is an elliptic curve over $F$, and $E^{\prime}[2] \cong E[2]$ as $G_{F}$-modules, then $E^{\prime}$ is isomorphic to $\mathcal{E}_{u, v}$ for some $u, v \in F$. Conversely, if $u, v \in F$ and $\mathcal{E}_{u, v}$ is nonsingular, then $\mathcal{E}_{u, v}[2] \cong E[2]$ as $G_{F}$-modules,

$j\left(\mathcal{E}_{u, v}\right)=\frac{\left(3 a v^{2}+9 b u v-a^{2} u^{2}\right)^{3} j(E)}{27 a^{3}\left(v^{3}+a u^{2} v+b u^{3}\right)^{2}}, \quad$ and $\quad \Delta\left(\mathcal{E}_{u, v}\right)=3^{6}\left(v^{3}+a u^{2} v+b u^{3}\right)^{2} \Delta(E)$.

Corollary 2. Suppose $F$ is a field of characteristic different from 2 and 3, and $E: y^{2}=x^{3}+a x+b$ is an elliptic curve over $F$. Let

$$
C(u, v)=\frac{\left(3 a v^{2}+9 b u v-a^{2} u^{2}\right)^{3}}{27 a^{3}\left(v^{3}+a u^{2} v+b u^{3}\right)^{2}} .
$$

Suppose $E^{\prime}$ is an elliptic curve over $F$. If $j\left(E^{\prime}\right) \neq 0,1728$, and for some $(u, v) \in$ $\mathbf{P}^{1}(F)$ we have 
(i) $\frac{j\left(E^{\prime}\right)}{j(E)}=C(u, v) \quad$ if $a \neq 0, \quad$ or

(ii) $\frac{j\left(E^{\prime}\right)}{j(E)-1728}=\frac{-4 C(u, v) a^{3}}{27 b^{2}} \quad$ if $b \neq 0$,

then $E^{\prime}[2] \cong E[2]$. Conversely, if $E^{\prime}[2] \cong E[2]$, then there is a point $(u, v) \in \mathbf{P}^{1}(F)$ such that $j\left(E^{\prime}\right)$ satisfies (i) if $a \neq 0$ and (ii) if $b \neq 0$.

We thank the NSF and NSA for financial support. Silverberg thanks AIM and the UC Berkeley math department for their hospitality.

\section{PROOFs}

Lemma 3. Suppose $F$ is a field and $\varphi(x) \in F[x]$ is a polynomial with no multiple roots. Let $\Psi_{\varphi}$ denote the set of roots of $\varphi$.

(i) There is a $G_{F}$-equivariant bijection $\Psi_{\varphi} \stackrel{\sim}{\rightarrow} \operatorname{Hom}_{F-\text { algebra }}\left(F[x] /(f(x)), F^{\text {sep }}\right)$.

(ii) The F-algebra of $G_{F}$-equivariant maps from $\Psi_{\varphi}$ to $F^{\text {sep }}$ is isomorphic to $F[x] /(f(x))$.

Proof. Assertion (i) is clear, and (ii) follows from Lemma 5 on p. A.V.75 of [1].

Lemma 4. Suppose $E: y^{2}=f(x)$ and $E^{\prime}: y^{2}=g(x)$ are elliptic curves over a field $F$ with $f(x), g(x) \in F[x]$ of degree 3 . Then $E[2] \cong E^{\prime}[2]$ as $G_{F}$-modules if and only if $F[x] /(f(x)) \cong F[x] /(g(x))$ as $F$-algebras.

Proof. We apply Lemma 3 with $\varphi=f$ and $g$. Since the roots of $f$ are the $x$-coordinates of the elements of $E[2]-0$, there is a $G_{F}$-equivariant bijection $\Psi_{f} \stackrel{\sim}{\rightarrow} E[2]-0$. Similarly we have $\Psi_{g} \stackrel{\sim}{\rightarrow} E^{\prime}[2]-0$. Thus by Lemma $3, F[x] /(f(x)) \cong$ $F[x] /(g(x))$ as $F$-algebras if and only if $E[2]-0 \cong E^{\prime}[2]-0$ as $G_{F}$-sets. Since every bijection $E[2]-0 \stackrel{\sim}{\rightarrow} E^{\prime}[2]-0$ extends to a group isomorphism $E[2] \stackrel{\sim}{\rightarrow} E^{\prime}[2]$, the lemma follows.

Proof of Theorem 1. Write $f(x)=x^{3}+a x+b$, so $E$ is the elliptic curve $y^{2}=f(x)$, and let $E^{\prime}$ be an elliptic curve $y^{2}=g(x)=x^{3}+\alpha x+\beta$ with $\alpha, \beta \in F$.

Suppose $E[2] \cong E^{\prime}[2]$ as $G_{F}$-modules. By Lemma 4 , there is an isomorphism of $F$-algebras $\phi: F[z] /(g(z)) \stackrel{\sim}{\rightarrow} F[x] /(f(x))$. Let $\phi(z)=3 u x^{2}+3 v x+w$ with $u, v, w \in F$. (The extra factors of 3 remove denominators which would otherwise occur in the equation for $\mathcal{E}_{u, v}$ and the formulas below.) The trace of $\phi(z)$ acting by multiplication on $F[x] /(f(x))$ is $3 w-6 a u$, but the trace of $z$ acting by multiplication on $F[z] /(g(z))$ is zero. Since $\phi$ is an isomorphism, we must have $w=2 a u$. Using this, the characteristic polynomial of $\phi(z)$ acting on $F[x] /(f(x))$ is

$h(T)=T^{3}+3\left(3 a v^{2}+9 b u v-a^{2} u^{2}\right) T+27 b v^{3}-18 a^{2} u v^{2}-27 a b u^{2} v-\left(2 a^{3}+27 b^{2}\right) u^{3}$.

Again, since $\phi$ is an isomorphism, we conclude that $h(T)=g(T)$, i.e., $E^{\prime}$ is $\mathcal{E}_{u, v}$ as desired.

Conversely, suppose that $u, v \in F$ are such that

$$
\alpha=3\left(3 a v^{2}+9 b u v-a^{2} u^{2}\right), \quad \beta=27 b v^{3}-18 a^{2} u v^{2}-27 a b u^{2} v-\left(2 a^{3}+27 b^{2}\right) u^{3} .
$$

Then working backwards through the argument above, one can show that the map $z \mapsto 3 u x^{2}+3 v x+2 a u$ induces a homomorphism $\phi: F[z] /(g(z)) \rightarrow F[x] /(f(x))$. The determinant of $\phi$ with respect to the bases $\left\{1, z, z^{2}\right\}$ and $\left\{1, x, x^{2}\right\}$ is $27\left(v^{3}+\right.$ $\left.a u^{2} v+b u^{3}\right)$. However, the discriminant of $g$ is $3^{6}\left(4 a^{3}+27 b^{2}\right)\left(v^{3}+a u^{2} v+b u^{3}\right)^{2}$. Since $E^{\prime}$ is an elliptic curve, the discriminant of $g$ must be nonzero, and hence the 
determinant of $\phi$ is nonzero so $\phi$ is an isomorphism. By Lemma 4 , it follows that $E[2] \cong E^{\prime}[2]$ as $G_{F}$-modules.

The formulas for the $j$-invariant and the discriminant are immediate.

Proof of Corollary 2. If $u, v \in F$ are such that $j\left(E^{\prime}\right)$ satisfies (i) or (ii), then $\mathcal{E}_{u, v}$ is nonsingular (by the computation of its discriminant in Theorem 1) and $j\left(E^{\prime}\right)=$ $j\left(\mathcal{E}_{u, v}\right)$. If $j\left(E^{\prime}\right) \neq 0,1728$, then $E^{\prime}$ is a quadratic twist of $\mathcal{E}_{u, v}$. Therefore using Theorem 1 , we have $E^{\prime}[2] \cong \mathcal{E}_{u, v}[2] \cong E[2]$. Conversely, if $E^{\prime}[2] \cong E[2]$, then by Theorem 1 we can find $u, v \in F$ such that $E^{\prime} \cong \mathcal{E}_{u, v}$. By Theorem 1 we have (i) and (ii).

\section{A DIFFERENT METHOD}

Applying the method of [3] (see also $\S 3$ of [5]) to the case $N=2$, one again obtains explicit equations for the family of elliptic curves with mod 2 representation isomorphic to that of $E$. We show below how the algorithm works in this case. Suppose $F$ is a field with $\operatorname{char}(F) \neq 2,3$, and $E: y^{2}=x^{3}+a x+b$ is an elliptic curve over $F$. Note that mod 2 representations do not change under quadratic twist. Every elliptic curve $E^{\prime}$ over $F$ such that the $G_{F}$-action on $E^{\prime}[2]$ is trivial is a quadratic twist of

$$
A_{\lambda}: y^{2}=x(x-1)(x-\lambda)
$$

with $\lambda \in F-\{0,1\}$. Putting $A_{\lambda}$ in Weierstrass form we obtain

$$
E_{\lambda}: y^{2}=x^{3}+a_{4}(\lambda) x+a_{6}(\lambda),
$$

where

$$
a_{4}(\lambda)=-\frac{1}{3}\left(\lambda^{2}-\lambda+1\right), \quad a_{6}(\lambda)=-\frac{1}{27}\left(2 \lambda^{3}-3 \lambda^{2}-3 \lambda+2\right) .
$$

The algorithm in $\S 3$ of [3] shows that the equations we are looking for are of the form

$$
d y^{2}=x^{3}+a(t) x+b(t)
$$

with

$$
d \in F, \quad a(t)=\mu^{-2}(\gamma t+1)^{2} a_{4}(A(t)), \quad \text { and } \quad b(t)=\mu^{-3}(\gamma t+1)^{3} a_{6}(A(t)),
$$

where $u_{0}$ satisfies $j\left(E_{u_{0}}\right)=j(E), \mu$ satisfies

$$
a_{4}\left(u_{0}\right)=a \mu^{2} \quad \text { and } \quad a_{6}\left(u_{0}\right)=b \mu^{3},
$$

and

$$
A(t)=\frac{\alpha t+u_{0}}{\gamma t+1}
$$

with $\alpha$ and $\gamma$ chosen so that $a(t), b(t) \in F[t]$.

If $a b \neq 0$, let $j=j(E)$ and let $u_{0}$ be a root of the numerator (as a polynomial in $\lambda$ ) of

$$
\begin{aligned}
& j\left(E_{\lambda}\right)-j= \\
& \frac{256-768 \lambda+(1536-j) \lambda^{2}+(2 j-1792) \lambda^{3}+(1536-j) \lambda^{4}-768 \lambda^{5}+256 \lambda^{6}}{\lambda^{2}(\lambda-1)^{2}} .
\end{aligned}
$$

Let

$$
\mu=\frac{a_{6}\left(u_{0}\right) a}{a_{4}\left(u_{0}\right) b}=\frac{\left(2 u_{0}^{3}-3 u_{0}^{2}-3 u_{0}+2\right) a}{9\left(u_{0}^{2}-u_{0}+1\right) b} \in\left(F^{\mathrm{sep}}\right)^{\times},
$$




$$
\alpha=\frac{3\left(u_{0}-2\right) \mu^{3} b}{u_{0}\left(u_{0}-1\right)}, \quad \gamma=\frac{3\left(2 u_{0}-1\right) \mu^{3} b}{u_{0}\left(u_{0}-1\right)} \quad \in F^{\text {sep }} .
$$

With these values, equation (1) becomes

$$
d y^{2}=x^{3}+a\left(1+(J-1) t^{2}\right) x+b\left(1+3 t-3(J-1) t^{2}-(J-1) t^{3}\right),
$$

where

$$
J=\frac{j(E)}{1728}=\frac{4 a^{3}}{4 a^{3}+27 b^{2}} .
$$

For $d \in F$ and $t \in \mathbf{P}^{1}(F)$, this gives the elliptic curves over $F$ with mod 2 representation isomorphic to that of $E$, when $a b \neq 0$.

Similarly, if $b=0$, then

$$
j\left(E_{\lambda}\right)-j(E)=\frac{64(-2+\lambda)^{2}(1+\lambda)^{2}(-1+2 \lambda)^{2}}{(-1+\lambda)^{2} \lambda^{2}} .
$$

With $u_{0}=2, \mu=1 / \sqrt{-a}, \alpha=0$, and $\gamma=3 \sqrt{-a}$, equation (1) becomes

$$
d y^{2}=x^{3}+a\left(1-3 a t^{2}\right) x+2 a^{2} t\left(1+a t^{2}\right) .
$$

If $a=0$, then

$$
u_{0}=\frac{1+\sqrt{-3}}{2}, \quad \mu=\frac{-1}{b^{1 / 3} \sqrt{-3}}, \quad \alpha=\frac{b^{1 / 3}(1-\sqrt{-3})}{2}, \quad \text { and } \quad \gamma=b^{1 / 3}
$$

yield the equation

$$
d y^{2}=x^{3}+3 b t x+b\left(1-b t^{3}\right) .
$$

\section{REFERENCES}

[1] N. Bourbaki, Algebra II, Springer, Berlin, 1990.

[2] B. Mazur, Rational isogenies of prime degree, Invent. Math. 44 (1978), 129-162.

[3] K. Rubin, A. Silverberg, Families of elliptic curves with constant mod $p$ representations, in Conference on Elliptic Curves and Modular Forms, Hong Kong, December 18-21, 1993, Intl. Press, Cambridge, Massachusetts, 1995, pp. 148-161.

[4] _ Mod 6 representations of elliptic curves, To appear in Proc. Symp. Pure Math., AMS, Providence.

[5] A. Silverberg, Explicit families of elliptic curves with prescribed mod $N$ representations, in Modular Forms and Fermat's Last Theorem, eds. Gary Cornell, Joseph H. Silverman, Glenn Stevens, Springer, Berlin (1997), 447-461.

Department of Mathematics, Stanford University, Stanford, CA,

Department of Mathematics, Ohio State University, Columbus, Ohio 43210

E-mail address: rubin@math.stanford.edu

Department of Mathematics, Ohio State University, 231 W. 18 Avenue, Columbus, Оніо 43210

E-mail address: silver@math.ohio-state.edu 